\title{
STOCHASTIC EVOLUTION OF COSMOLOGICAL PARAMETERS IN THE EARLY UNIVERSE
}

\author{
C. Sivakumar ${ }^{\dagger}$, Moncy V. John ${ }^{\ddagger}$ and K. Babu Joseph \\ Department of Physics, Cochin University of Science and Technology \\ Kochi 682022, India
}

Short title : Stochastic Approach To Early Universe

\begin{abstract}
We develop a stochastic formulation of cosmology in the early universe, after considering the scatter in the redshift-apparent magnitude diagram in the early epochs as an observational evidence for the non-deterministic evolution of early universe. We consider the stochastic evolution of density parameter in the early universe after the inflationary phase qualitatively, under the assumption of fluctuating $w$ factor in the equation of state, in the Fokker-Planck formalism. Since the scale factor for the universe depends on the energy density, from the coupled Friedmann equations we calculated the two variable probability distribution function assuming a flat space geometry.
\end{abstract}

Key words. Cosmology; stochastic equations; Fokker-Planck equation.

PACS Nos 98.80; 02.50

$\dagger$ e-mail: sivakumarc @ cusat. ac. in

† Permanent Address: Department of Physics, St. Thomas College,

Kozhencherri, Kerala, India 689641 e-mail: moncy @ stthom. ernet. in 


\section{Introduction}

The simplest model of the universe [1], called the Friedmann model or hot big bang model, is based on the assumption that the matter distribution in the universe is homogeneous and isotropic on very large scales (cosmological principle). This assumption simplifies the problem of solving Einstein's equations. The assumption of homogeneity leads to the prediction of deterministic Hubble's law and one expects a scatter free redshift-magnitude diagram for galaxies and other extra galactic objects. For nearer galaxies, Hubble's law is justified by a relatively scatter-free redshift-magnitude relation. This corresponds to a deterministic evolution of the universe. However, for high redshift quasars (in the Hewitt-Burbidge catalogue [2,3]) and for the latest Type Ia supernovae data $[4,5]$, the redshift-magnitude diagram is a scatter diagram, i.e., they are in contrast with the deterministic Hubble's law. If Hubble's law is valid for all extra-galactic objects, then the evolution of the universe (or expansion rate) is nondeterministic in the early epoch, as indicated by the scatter diagram. We observe that the scatter increases as we probe into more and more distant epochs. The small scatter for galaxies at low redshifts is explained as due to peculiar velocities and the conventional explanation for the peculiar velocities is that they are induced by the observed density perturbations. This may be adequate to account for the observed peculiar velocities of objects, in the range of $100 \mathrm{~km} / \mathrm{s}-400 \mathrm{~km} / \mathrm{s}$. Thus this may lead to a very small scatter at low redshifts. But since the amplitude of density perturbations in the early universe was very low, the large scatter at very high redshifts 
remains unexplained and it is desirable to look for some alternative mechanism. Some authors speculate $[3,6]$ that the quasars are not at their cosmological distances and have proposed some non-cosmological contributions to $z$ as a possible explanation for the scatter in the quasar data. Since the distance measurements are extremely difficult for quasars, due to the difficulty in identifying standard candles, the scatter may be caused by the variation of intrinsic luminosities of quasars of same $z$. However, the data is more accurate for supernovae and the scatter in its redshift-magnitude diagram is not due to either peculiar velocities or variation of intrinsic luminosities. In [7], we proposed that a fluctuating equation of state or a fluctuating mean $w$ factor in the equation of state led to a non-deterministic or stochastic Hubble parameter and argued that such a fluctuating expansion rate in the early universe might have led to a randomness in the recession velocities of objects, in addition to peculiar velocities and will produce a scatter in the redshift-magnitude diagram in those epochs. Here

we develop a more general description of the stochastic dynamics of the early universe, and discuss the non-deterministic character of total density of the universe, as well as the scale factor for the universe. Such a stochastic approach is necessary, when the mean $w$ factor in the equation of state of the cosmic fluid is a fluctuating quantity.

\section{Observational issues and assumptions}

In this section, we state the conditions under which a stochastic equation of state (or a fluctuating mean $w$ factor) emerges. In standard cosmology, the cosmological 
fluid is in fact not unicomponent, instead matter and radiation (with equation of state $p_{m}=0$ and $p_{r}=\left(\frac{1}{3}\right) \rho_{r}$, respectively) in disequilibrium coexist in many 'elementary subvolumes' of the universe [8]. Some recent measurements on the age of the universe, Hubble parameter, deceleration parameter, gravitational lensing etc., point to the need of extending the standard model by including some new energy density (missing energy) in the present universe, in addition to the usual relativistic/non-relativistic energy density. Recent observations using Type Ia supernovae [4,5] as standard candles seem to indicate that the universe may be accelerating, driven by a positive cosmological constant (or a vacuum energy density $\rho_{v}$ ) with the equation of state $p_{v}=-\rho_{v}$. Some authors $[9,10]$ introduced Quintessence or Q-component (for exa: scalar fields rolling down a potential with negative pressure or cosmic strings etc.) with equation of state $p_{Q}=w_{Q} \rho_{Q},-1<w_{Q} \leq 0$, in addition to matter density. The case for a positive cosmological constant has been considered very often in the literature [11-23]. All these models favour a flat universe with $\Omega=\Omega_{m}+\Omega_{v}+\cdots=1$, where $\Omega$ is the ratio of energy density to critical density. Thus our universe may be approximated by a perfect fluid having many components, each with equation of state $p_{i}=w_{i} \rho_{i},-1 \leq w_{i} \leq+1$, $i=1,2 \cdots$. If we denote the total energy density due to all such components as $\rho$, then

$$
\rho=\rho_{m}+\rho_{r}+\rho_{v}+\cdots
$$

where $\rho_{m}, \rho_{r}, \rho_{v}$ etc. are the average densities of matter, radiation, vacuum energy etc. 
In a similar way the total pressure $p$ can be written as

$$
p=p_{m}+p_{r}+p_{v}+\cdots
$$

In general, $\rho=\sum \rho_{i}$, where $\rho_{i}$ 's represent energy densities of various components. From the energy-momentum conservation law (here it is assumed that only the total energy density is conserved), we have

$$
\dot{\rho}=-3 \frac{\dot{a}}{a}(\rho+p)=-3 \frac{\dot{a}}{a} \rho(1+w),
$$

where $p=\sum p_{i}$ is the total pressure, and the ratio $w=p / \rho$ should lie between -1 and +1 . Splitting $\rho$ and $p$ into individual components, the above equation becomes

$$
\dot{\rho}_{1}+\dot{\rho}_{2}+\dot{\rho}_{3}+\cdots \cdot=-3 \frac{\dot{a}}{a}\left[\rho_{1}\left(1+w_{1}\right)+\rho_{2}\left(1+w_{2}\right)+\rho_{3}\left(1+w_{3}\right)+\cdot \cdot\right] .
$$

From Eqs. (3) and (4), we get

$$
w(t)=-\frac{\left[\dot{\rho}_{1}+\dot{\rho}_{2}+\cdots\right]}{3(\dot{a} / a) \rho}-1=\frac{\left[\rho_{1}\left(1+w_{1}\right)+\rho_{2}\left(1+w_{2}\right)+\cdots \cdot \cdot\right]}{\rho}-1 .
$$

Here we assumed that the total energy density $\rho$ is conserved and not the parts corresponding to $\rho_{1}, \rho_{2} \cdots$ separately, and hence there can be creation of one component at the expense of other components and since $\dot{a} / a$ and $\rho$ are large in the earlier epochs, at least some of the $\dot{\rho}_{i}$ 's will be significantly large in this period. Since recent observations indicate the existence of vacuum energy even in the present universe, one can expect that particle creation continued for a fairly long period in the early universe [24]. If we 
have a many component fluid, then the Einstein equations, along with the equations of state of individual components, are insufficient to determine the creation rates of each component. In [25], Weinberg discusses the consequences of the presence of a cosmological constant (vacuum energy) in the energy density. He discusses some phenominological proposals made by some authors, of the energy transfer between vacuum and matter or vacuum and radiation, in such a way that either $\rho_{v} / \rho_{m}$ or $\rho_{v} / \rho_{r}$ remains constant, respectively. He also considers the possibility of creation of radiation from vacuum energy, keeping $\rho_{v} / \rho_{m}$ fixed. However, in a general case as in Eq. (5) above, one cannot expect any kind of creation in the universe to be a smooth process, since they can be sporadic events occurring in different locations and times, like those occurring in galactic nuclei. Here, as in the case of other stochastic processes like Brownian motion, a complete solution of the macroscopic system (universe) would consist in solving all the microscopic equations describing the creation processes, but such a rigorous derivation will be very complicated or even impossible. In this context, a stochastic approach is more reasonable, in which we consider the creation rates to be fluctuating, leading to fluctuations in the ratios $\rho_{i} / \rho$. As it clear from Eq. (5), this in turn, will lead to a fluctuating $w$ factor, which is the key assumption made in this paper. Such a fluctuating quantity modifies the dynamics of the early universe, where the evolution of the cosmological parameters, like the total energy density $(\rho)$ and scale factor for the universe $(a)$ becomes stochastic or non-deterministic. The fluctuations in the ratio $\rho_{i} / \rho$, that we are taking in to account here are classical, i.e., our stochastic model is a 
modification to the classical Friedmann model of the early universe, when fluctuations are significant. In [22], Fang et al discuss a stochastic approach to early universe (before recombination epoch), when cosmic fluid consisting of primeval plasma and radiation, is not perfect, but have dissipations due to differences in the adiabatic cooling rates of the components of the fluid and the possible energy transfer between them. Physically motivated interaction models are also proposed in the literature [26,27], which lead to energy transfer between various components. However, once we probe into still earlier epochs (stages of inflation etc.), quantum fluctuations become very important. Many authors discuss the need for a stochastic approach to inflation $[28,29,30]$, when the quantum fluctuations of the scalar field are significant, and try to get a probability distribution function for the scalar field after solving the quantum Langevin equation (or FPE) describing the evolution of the scalar field. However we adopt the stochastic approach in the classical regime, where fluctuations in the creation rates and also in the possible energy transfer between different components of the cosmic fluid lead to a stochastic equation of state. This causes a non-deterministic (stochastic) expansion rate for the universe, described by a set of stochastic differential equations, instead of the deterministic Friedmann equations and we evaluate the probability distribution function of the cosmological parameters. In section 3 we discuss the stochastic character of the density parameter of the universe on the basis of Fokker-Planck formalism [31] . In section 4 we analyse the effect of such phenomena on the expansion factor for the universe and a two variable distribution function is derived. 


\section{Stochastic evolution of density parameter}

Suppose the universe is approximated by a many component fluid in the early epochs, with a fluctuating $w$ term in the equation of state. Now we write the evolution equation for the total density in the early universe (assuming that, total energy density is conserved), immediately after inflation, when curvature factor appearing in the field equation is negligible, so that the background is approximately flat.

$$
\dot{\rho}=-3 \frac{\dot{a}}{a}[1+w(t)] \rho .
$$

Overdots denote time derivatives. Using Friedmann equations we have

$$
\dot{\rho}=-\sqrt{24 \pi G}[1+w(t)] \rho^{3 / 2} .
$$

Above equation is a stochastic differential equation of the Langevin type. Since $w$ is a fluctuating 'force' term, $\rho$ is a stochastic variable or its evolution is non-deterministic. The random behaviour of $\rho$ in the early universe is due to fluctuations in the factor $w$ alone. If fluctuations are zero we are back to the deterministic standard model. We apply stochastic methods [31] for the analysis of the above equation and the probability distribution function is calculated using Fokker-Planck formalism. By making use of the transformation

$$
\sigma=\frac{1}{(6 \pi G \rho)^{1 / 2}},
$$

eq. (7) gets modified into

$$
\dot{\sigma}=1+w(t)
$$


which is a non-deterministic, stochastic first order differential equation (Here $\sigma \propto t$ for a pure deterministic case). To solve eq. (9) we use certain simplifying assumptions that the fluctuating factor $w$ is Gaussian $\delta$-correlated, with mean zero. Though these assumptions are taken for the sake of simplicity, we expect that they are reasonable when compared to the time scales involved.

If we have a general Langevin type equation of the form

$$
\dot{y}=h(y, t)+g(y, t) \Gamma(t),
$$

where $\Gamma(t)$ is a fluctuating quantity with zero mean and Gaussian $\delta$-correlated, then the corresponding Fokker-Planck equation (FPE) describing the time evolution of the probability distribution function $W(y, t)$ can be written as

$$
\frac{\partial W(y, t)}{\partial t}=\sum_{n=1}^{\infty}\left(-\frac{\partial}{\partial y}\right)^{n} D^{(n)}(y) W(y, t)
$$

where $D^{(n)}(y)$ are the Kramers Moyal expansion coefficients given by

$$
D^{(n)}(y, t)=\frac{1}{n !}\left[\lim _{\tau \rightarrow 0} \frac{1}{\tau}\left\langle[y(t+\tau)-x]^{n}\right\rangle\right]_{y(t)=x} .
$$

Here $y(t+\tau)(\tau>0)$ is a solution of eq. (10) which at time $t$ has the sharp value $y(t)=x$. Under the assumption of $\delta$-correlation and zero mean of $\Gamma(t)$, all coefficients vanishes for $n \geq 3$ and retain only the coefficients $D^{(1)}$ and $D^{(2)}$, called drift and diffusion coefficients respectively. Following the standard procedure [31], eq. (9) leads to the FPE

$$
\frac{\partial W(\sigma, t)}{\partial t}=-\frac{\partial W}{\partial \sigma}+D \frac{\partial^{2} W}{\partial \sigma^{2}}
$$


Here we used drift coefficient $D^{(1)}=1$ and diffusion coefficient $D^{(2)}=D(D$ is a constant with dimension of time, which is introduced for the purpose of generality). In order to obtain non-stationary solutions of eq. (13) we use a separation ansatz for $W(\sigma, t)$

$$
W(\sigma, t)=\phi(\sigma) e^{-\lambda t}
$$

Substituting this into eq. (13) and solving for $\phi(\sigma)$ we get

$$
\phi(\sigma)=A \exp \left[\frac{\sigma}{2 D}+i k \sigma\right]
$$

where

$$
k= \pm \sqrt{\frac{\lambda}{D}-\frac{1}{4 D^{2}}} .
$$

Thus we see that for $\lambda<1 / 4 D, k^{2}$ is negative and the solution is exponentially diverging, which is not a physically reasonable solution. Hence we conclude that $\lambda \geq 1 / 4 D$, so that $k$ is real. We write the most general solution as

$$
W(\sigma, t)=\sum_{n} c_{n} \phi_{n}(\sigma) e^{-\lambda_{n} t}
$$

where $c_{n}$ can be real or complex but $W(\sigma, t)$ is always real. For a continuous parameter $k$, from eqs. (14) and ( 15) the general solution or the distribution function is given by

$$
W(\sigma, t)=A \int_{-\infty}^{+\infty} \exp \left[\frac{\sigma}{2 D}+i k \sigma-k^{2} D t-\frac{t}{4 D}\right] d k
$$

We choose $A=1 / 2 \pi$ for normalization purpose. On evaluating the integral we find the distribution function

$$
W(\sigma, t)=\frac{1}{\sqrt{4 \pi D t}} \exp \left[-\frac{(\sigma-t)^{2}}{4 D t}\right],
$$


which is in Gaussian form. The expectation value of the stochastic variable $\sigma$ is $\langle\sigma\rangle=t$ and corresponds to the deterministic solution of eq. (9). The width of the Gaussian is

found from the variance, $v=\left\langle(\sigma-\langle\sigma\rangle)^{2}\right\rangle=2 D t$. Once $W(\sigma, t)$ is known it is straight forward to write the distribution function $W(\rho, t)$ as

$$
W(\rho, t)=\frac{1}{\sqrt{96 \pi^{2} D G \rho^{3} t}} \exp \left[-\frac{(1-t \sqrt{6 \pi G \rho})^{2}}{24 \pi G D \rho t}\right] .
$$

We can also find the transition probability for the stochastic variable to change from an initial state $\left(\sigma^{\prime}, t^{\prime}\right)$ to a final state $(\sigma, t)$ as

$$
P\left(\sigma, t \mid \sigma^{\prime}, t^{\prime}\right)=\frac{1}{\sqrt{4 \pi D\left(t-t^{\prime}\right)}} \exp \left[-\frac{\left[\left(\sigma-\sigma^{\prime}\right)-\left(t-t^{\prime}\right)\right]^{2}}{4 D\left(t-t^{\prime}\right)}\right]
$$

with the initial value

$$
P\left(\sigma, t \mid \sigma^{\prime}, t\right)=\delta\left(\sigma-\sigma^{\prime}\right)
$$

indicating Markovian nature of the random variable $\sigma$. In terms of $\rho$ eq. (21) becomes

$$
P\left(\rho, t \mid \rho^{\prime}, t^{\prime}\right)=\frac{1}{\sqrt{4 \pi D\left(t-t^{\prime}\right)}} \exp \left[-\frac{\left[\left(\sqrt{\rho^{\prime}}-\sqrt{\rho}\right)-\sqrt{6 \pi G \rho \rho^{\prime}}\left(t-t^{\prime}\right)\right]^{2}}{24 \pi G \rho \rho^{\prime} D\left(t-t^{\prime}\right)}\right]
$$

This represent the probability for the energy density to change from an initial value $\rho$ to a final value $\rho^{\prime}$ during a time interval $\left(t-t^{\prime}\right)$ in the early epochs. This characterises the stochastic behaviour of density evolution in the early universe.

\section{Scale factor as a stochastic variable}

Under the assumption that the factor $w$ is fluctuating during the early epochs, the evolution of the scale factor also becomes non-deterministic, since the time evolution 
of $a(t)$ is determined by the total density. So we have a system of coupled stochastic differential equations derived from Friedmann equations

$$
\dot{a}=\sqrt{\frac{8 \pi G}{3} \rho,}
$$

and

$$
\dot{\rho}=-\sqrt{24 \pi G}[1+w(t)] \rho^{3 / 2} .
$$

Here we are considering the dynamics of the universe immediately after inflation, so that the background can be treated approximately flat. With the transformation defined in eq. (8), the above system of equations reduce to

$$
\dot{a}=\frac{2 a}{3 \sigma},
$$

and

$$
\dot{\sigma}=1+w(t)
$$

Following the standard procedure [31] we have the drift coefficients $D_{a}^{(1)}=\frac{2 a}{3 \sigma}, D_{\sigma}^{(1)}=1$ and the diffusion coefficient $D_{\sigma \sigma}^{(2)}=D$ is assumed to be a constant. It shall be noted that this diffusion term arises due to fluctuations in $w$ alone. The two variable FPE for the distribution function $W(a, \sigma, t)$ can be written as

$$
\frac{\partial W}{\partial t}=-\frac{2}{3 \sigma}\left[W+a \frac{\partial W}{\partial a}\right]-\frac{\partial W}{\partial \sigma}+D \frac{\partial^{2} W}{\partial \sigma^{2}} .
$$

We can solve the FPE by first assuming the ansatz

$$
W(a, \sigma, t)=U(a) V(\sigma) e^{-\lambda t}
$$


and substituting into eq. (28). We obtain

$$
\frac{\sigma}{V} D \frac{d^{2} V}{d \sigma^{2}}-\frac{\sigma}{V} \frac{d V}{d \sigma}+\lambda \sigma=\frac{2}{3}\left[\frac{a}{U} \frac{d U}{d a}+1\right] .
$$

Each side in this equation can be equated to a constant $m$. When $m=0$

$$
U(a) \propto \frac{1}{a}
$$

and

$$
V(\sigma) \propto \exp \left[\frac{\sigma}{2 D}+i k \sigma\right]
$$

with $k$ given by eq. (16). A physically reasonable solution exists for $\lambda \geq 1 / 4 D$, which is

$$
W(a, \sigma, t)=\frac{B}{a} \exp \left[\frac{\sigma}{2 D}+i k \sigma-\lambda t\right]
$$

Here $B$ is a normalization constant, chosen to be $1 / 2 \pi$. One point to be noted is the most general solution to eq. (30) when $m \neq 0$, is a series solution owing to the singularity at $\sigma=0$. One can find a limiting solution as $\sigma \rightarrow 0$, in the following form

$$
W(a, \sigma) \longrightarrow a^{\left(\frac{2}{3} m-1\right)} \frac{\exp \left(\frac{\sigma}{2 D}\right)}{(m / D)} \sum_{n=1}^{\infty} \frac{\left(\frac{m}{D} \sigma\right)^{n}}{n !(n-1) !}
$$

However we will get a real general solution in a compact form after integrating eq. (33) in the range $-\infty<k<+\infty$

$$
W(a, \sigma, t)=\frac{1}{\sqrt{4 \pi D t}}(a)^{-1} \exp \left[-\frac{(\sigma-t)^{2}}{4 D t}\right] .
$$

In terms of $\rho$, it becomes 


$$
W(a, \rho, t)=\frac{1}{\sqrt{96 \pi^{2} D G \rho^{3} a^{2} t}} \exp \left[-\frac{(1-t \sqrt{6 \pi G \rho})^{2}}{24 \pi G D \rho t}\right] .
$$

The two variable probability distribution function is Gaussian in $\sigma$, and diverges as $a \rightarrow 0$, where classical approach fails and quantum theory takes over. Now we write the expression for the transition probability

$$
P\left(a, \sigma, t \mid a^{\prime}, \sigma^{\prime}, t^{\prime}\right)=\frac{\left(a a^{\prime}\right)^{-1}}{\sqrt{4 \pi D\left(t-t^{\prime}\right)}} \exp \left[-\frac{\left[\left(\sigma-\sigma^{\prime}\right)-\left(t-t^{\prime}\right)\right]^{2}}{4 D\left(t-t^{\prime}\right)}\right]
$$

In terms of $\rho$ and $a$ it becomes

$$
P\left(a, \rho, t \mid a^{\prime}, \rho^{\prime}, t^{\prime}\right)=\left(a a^{\prime}\right)^{-1} \frac{\exp \left[-\frac{\left[\left(\sqrt{\rho^{\prime}}-\sqrt{\rho}\right)-\sqrt{6 \pi G \rho \rho^{\prime}}\left(t-t^{\prime}\right)\right]^{2}}{24 \pi G \rho \rho^{\prime} D\left(t-t^{\prime}\right)}\right]}{\sqrt{4 \pi D\left(t-t^{\prime}\right)}},
$$

which represents the transition probability for the variables to change from the state $\left(a^{\prime}, \rho^{\prime}\right)$ to $(a, \rho)$. Thus the scale factor $a$ together with the density $\rho$ evolves in a nondeterministic way, which in turn strongly influence the formation of large scale structure in the universe, since the evolution of the density perturbations are also depend on $w$. In all these cases we get Gaussian distributions, which is sharply peaked initially but spread out with time.

\section{Conclusion}

In the preceding sections we have described a stochastic approach to cosmology as a modification to the deterministic evolution of the universe in the standard model. In section 2, we have shown that fluctuations in the creation rates are physical processes 
which can lead to a stochastic equation of state. A fluctuating $w$ factor, in turn, will lead to fluctuations in the time - evolution of the energy density of the universe, as well as in the expansion factor for the universe. Thus both parameters become stochastic quantities, instead of remaining deterministic variables. A fluctuating $w$ factor will also lead to fluctuations in the time-evolution of the Hubble parameter; i.e., the expansion rate of the universe becomes a stochastic quantity, instead of being a deterministic variable. We predict that such a stochastic expansion rate might have led to a randomness in the recession velocities of objects and will produce a scatter in the Hubble diagram. Using the redshift-magnitude data $\mu_{0}$ for the Type Ia supernovae of Perlmutter et al [4], which corresponds to their Fit C, and the technique used in Riess et al [5], we have computed the probability distribution function $p\left(H_{0} \mid \mu_{0}\right)$ for a flat universe and compared it with the theoretical distribution [7], showing that both curves agree very well with $D=3.77 \times 10^{13} \mathrm{~s}$ and a half width of 0.011 . A similar estimate is possible also with the distribution function of the density parameter, provided we are given a known data for the density parameter. Also if we have some explicit examples of models where a stochastic $w$ emerges, the predicted value of $D$ may be compared with our estimation, but in this paper, we have not made any attempts in this regard. Thus, if $w$ is a fluctuating quantity, the evolution of the early universe becomes stochastic or non-deterministic (the scatter in the Hubble diagram indicates this), and the dynamical equations of those epochs are Langevin type equations, where one can evaluate the probability distribution functions of the variables. In [23], Berera 
and Fang describe dynamically how, the stochastic fluctuations arising from various dissipations, generate seeds of density perturbations in the early universe, apart from the quantum fluctuations of the standard inflationary model. Here we attempted to make a stochastic approach to the early universe due to fluctuations in the mean equation of state, which is a classical phenomenon. Since the stochastic equation of state, lead to fluctuations in the time - evolution of the total density of the universe, the density contrast will also be fluctuating. We propose to undertake this study in detail in a future publication.

To conclude, we note that the stochastic approach presented above is a modification to the standard model, when fluctuations are present. We have formulated a stochastic model and developed a set of non-deterministic, Langevin equations for the cosmological parameters in those epochs, under the assumption that the factor $w(t)$ is a fluctuating quantity, when the universe is approximated by a many component fluid. It is expected that diffusion coefficient is a crucial factor in the evolution of the universe, especially in the early phase, where it influences the time - evolution of density parameter and scale factor for the universe. As fluctuations die out with time $(D \rightarrow 0)$, the evolution becomes deterministic.

\section{Acknowledgements}

The authors thank the referee for valuable suggestions. One of us (CS) thanks CSIR, New Delhi for the award of a Research Fellowship. 


\section{References}

[1] P J E Peebles, Principles of Physical cosmology (Princeton series, Princeton, 1993)

[2] A Hewitt and G Burbidge, Astrophys. J. Supp. 63, 1 (1987)

[3] A K Kembhavi and J V Narlikar, Quasars and Active galactic Nuclei - An Introduction (Cambridge University press, Cambridge, 1999)

[4] S Perlmutter et al, Astrophys. J. 517, 565 (1999)

[5] A G Riess et al, Astron. J. 116, 1009 (1998)

[6] G R Burbidge, Extragalactic Energy sources edited by V K Kapathi (The Indian Academy of sciences, Bangalore, 1985) p. 87

[7] C Sivakumar, M V John and K Babu Joseph, Class. Quantum Gravit. (submitted)

[8] D Jou and D Pavon, Foundations of Big Bang Cosmology edited by F W Meyerstein (World Scientific, Singapore, 1987) p. 155

[9] R R Caldwell, R Dave and P J Steinhardt, Phys. Rev. Lett. 80, 1582 (1998)

[10] I Zlatev, L Wang and P J Steinhardt, Phys. Rev. Lett. 82, 896 (1999)

[11] L M Krauss, Astrophys. J. 501, 461 (1998)

[12] L M Krauss, Scientific American 280, 52 (1999)

[13] P J Steinhardt, Nature, 382, 768 (1996) 
[14] I Zehavi and A Dekel, Nature, 401, 252 (1999)

[15] G H Jacoby, Nature, 371, 741 (1994)

[16] V Sahni, Pramana-J. Phys. 53, 937 (1999)

[17] S Perlmutter et al, Astrophys. J. 483, 565 (1997)

[18] P J E Peebles, Nature, 398, 25 (1999)

[19] W L Freedman et al, Nature, 371, 757 (1994)

[20] M J Pierce et al, Nature, 371, 385 (1994)

[21] M Kamionkowski and N Toumbas, Phys. Rev. Lett. 77 , 587 (1996)

[22] L Z Fang, Z Huang and X P Wu, Int. J. of Mod. Phys. D5, 495 (1996)

[23] A Berera and L Z Fang, Phys. Rev. Lett. 72, 458 (1994)

[24] J C Carvalho, J A S Lima and I Waga, Phys. Rev. D46, 2404 (1992)

[25] S Weinberg, Rev. Mod. Phys., 61, 1 (1989)

[26] B Ratra and P J E Peebles, Phys. Rev. D37, 3407 (1988)

[27] P J E Peebles and B Ratra, Astrophys. J. 325, L17 (1988)

[28] M Mijic, Phys. Rev. D42, 2469 (1990)

[29] A A Starobinsky, Field theory Quantum Gravity and Strings edited by H J de Vega and N Sanchez (Springer, New York, 1986) p. 107 
[30] Y Nambu and M Sasaki, Phys. Lett. B219, 240 (1989)

[31] H Risken, The Fokker-Planck Equation (springer-Verlag, New York, 1984) 